\title{
Metacognitive Functioning in Individuals at Clinical High Risk for Psychosis
}

\author{
Mariapaola Barbato \\ University of Calgary, Canada \\ David L. Penn and Diana O. Perkins \\ University of North Carolina at Chapel Hill, USA
}

Scott W. Woods

Yale University, New Haven, USA

Lu Liu and Jean Addington

University of Calgary, Canada

\begin{abstract}
Background: Metacognition has been described as the knowledge of our own cognitive processes. Metacognitive deficits are common in schizophrenia, but little is known about metacognition before the onset of full-blown psychosis. Aims: This study aimed to longitudinally characterize metacognition in a sample of individuals at clinical high risk (CHR) for psychosis, and to determine if metacognition was related to later conversion to psychosis. Method: Participants (153 CHR individuals; 68 help seeking controls, HSC) were part of the large multi-site PREDICT study, which sought to determine predictors of conversion to psychosis. They were tested at baseline and 6 months using the MetaCognitions Questionnaire (MCQ) that has five sub-scales assessing different domains of metacognition. Results: Results of the mixed-effect models demonstrated significantly poorer scores at baseline for the CHR group compared to the HSC group in Negative beliefs about uncontrollability, Negative beliefs and the overall MCQ score. At the 6-month assessment, no difference was observed in metacognition between the two groups, but both groups showed improvement in metacognition over time. Those who later converted to psychosis had poorer performance on metacognitive beliefs at baseline. Conclusions: A poorer performance in metacognition can be seen as a marker of developing a full blown psychotic illness and confirms the potential value of assessing metacognitive beliefs in individuals vulnerable for psychosis.
\end{abstract}

Keywords: Clinical high risk, psychosis prodrome, metacognition, schizophrenia, helpseeking controls.

Reprint requests to Mariapaola Barbato, Mathison Centre for Mental Health Research and Education, 3280 Hospital Drive NW, Calgary AB T2N 4Z6, Canada. E-mail: mbarbato@ucalgary.ca 


\section{Introduction}

Metacognition is often described as "thinking about thinking" (e.g. Semerari et al., 2003) and refers to the ability to evaluate our own cognitive processing and to use these evaluations to guide behaviour. Metacognition is a multi-faceted concept that entails several different faculties, from being aware of one's mental processes and their fallibility, to controlling, interpreting and revising thinking and behaviour.

Some specific aspects of metacognition have been suggested to play a role in the development and maintenance of psychopathology (Wells and Matthews, 1994). In particular, Wells and Matthews (1996), in their Self-Regulatory Executive Function (S-REF) model, suggested that vulnerability to psychopathology is associated with an increase in self-focused attention, attentional bias and ruminative processing that generate dysfunctional beliefs. For example, individuals who develop problematic worrying (such as in generalized anxiety disorder) have both positive beliefs about the worry itself (e.g. "worrying helps me cope" or "I need to worry in order to remain organized"), and negative beliefs such as the loss of control of thoughts (e.g. "worrying thoughts enter my head against my will"). Both positive and negative metacognitive beliefs can influence behaviour to the point that maladaptive response styles such as rumination and thought suppression are developed and maintained over time, thus determining a vicious cycle that can lead to psychopathology. The S-REF model has been widely used to study the relationship between metacognition and psychiatric disorders and has been validated by numerous studies assessing metacognitive beliefs in emotional disorders (e.g. Cartwright-Hatton and Wells, 1997; Wells and Papageorgiou, 1998).

There is also interest in metacognition in schizophrenia. Building on the S-REF model, Morrison (2001) suggested that positive metacognitive beliefs about psychotic experiences are associated with the occurrence of hallucinations and delusions, and negative metacognitive beliefs about psychotic experiences are associated with distress in response to them. In other words, according to Morrison individuals with psychosis can hold positive beliefs about hallucinations such as "the voices are benevolent" or "the voices keep me company" and negative beliefs about hallucinations such as "the voices are dangerous" or "the voices are uncontrollable". These negative metacognitive beliefs can lead to an increase in arousal and safety-seeking behaviour, which in turn determines the occurrence of more hallucinations, thus establishing a vicious cycle. This model has been supported by studies showing that psychotic patients who experience auditory hallucinations exhibit dysfunctional metacognitive beliefs (i.e. higher scores in metacognition scales compared to healthy controls) (e.g. Morrison and Wells, 2003; Morrison, Nothard, Bowe and Wells, 2004; Morrison, Wells and Nothard, 2000). Furthermore, metacognitive dysfunction has been observed in samples of people who are at clinical high risk (CHR) for developing psychosis (Brett, Johns, Peters and McGuire, 2009; Morrison et al., 2002; Morrison, French and Wells, 2007) or have schizotypy (Barkus et al., 2010). Morrison et al. (2007) reported that individuals at CHR for psychosis score higher than non-patients on negative beliefs about thoughts and lower than psychotic patients on positive beliefs about worry, suggesting that negative beliefs about thoughts may play a role in determining vulnerability whereas positive beliefs about worry in a CHR group could potentially be a marker of conversion. Barkus and colleagues (2010) demonstrated by a comparison of a CHR sample with healthy volunteers that with increasing levels of schizotypy and high scores on the Launay-Slade Hallucination Scale (Launay and Slade, 1981), healthy volunteers showed similar dysfunctional metacognitive beliefs and 
distress to the CHR group, suggesting that individuals with high schizotypal traits are on a continuum with CHR individuals and that metacognitive impairment spreads across the psychosis continuum.

In a third study, Brett et al. (2009) compared individuals with psychosis, CHR individuals, individuals who had had at least 5 years psychotic-like experiences and healthy controls. Individuals with psychosis and those at CHR had poorer scores on the MCQ, particularly the negative beliefs subscale. However, most differences disappeared when anxiety and depression were controlled for.

Thus it seems as if dysfunctional positive and negative metacognitive beliefs already exist in those at clinical high risk of developing psychosis on the basis of experiencing subthreshold symptoms. However, samples have been small, cross-sectional and the role of metacognition and eventual conversion to psychosis has not been explored. The aim of this study was to assess metacognition longitudinally in a large sample of individuals at CHR of developing psychosis, to determine whether negative beliefs are prevalent in the CHR group, and if high scores in positive beliefs about worry may predict later conversion to psychosis.

\section{Method}

\section{Sample}

The sample consisted of 153 (88 males, 65 females) individuals at CHR of psychosis and 68 (40 males, 28 females) help-seeking controls (HSC). All of the participants were part of a multi-site NIMH funded study called "Enhancing the Prospective Prediction of Psychosis" (PREDICT). This was a 2-year longitudinal study conducted at the universities of Toronto, North Carolina, and Yale University to determine predictors of conversion in individuals at clinical high risk of developing psychosis; $58 \mathrm{CHR}$ and $28 \mathrm{HSC}$ were recruited at the Universities of Toronto, 59 CHR and 20 HSC at the University of North Carolina and 36 CHR and 20 HSC at Yale. All CHR individuals met the Criteria of Prodromal States (COPS) based on the Structured Interview for Prodromal Symptoms (SIPS) (McGlashan, Walsh and Woods, 2010). Among the CHR sample, 149 CHR participants met attenuated positive symptom syndrome (APSS) criteria, which includes the emergence or worsening of a non-psychotic level disturbance in thought content, thought process or perceptual abnormality over the past year, 2 participants met criteria for genetic risk and deterioration (GRD), which required either a first degree relative with a psychotic disorder or the subject having schizotypal personality disorder (SPD) plus at least a 30\% drop in functioning on the General Assessment of Functioning (GAF) scale in the past 12 months, and 2 participants met both APSS and GRD. The help-seeking control (HSC) group were individuals who had responded to recruitment efforts for the CHR sample, and appeared likely to meet prodromal criteria on a phone screen but after the initial comprehensive interview did not. The HSC group consisted of individuals with long standing symptoms i.e. attenuated positive symptoms had been present for more than one year $(n=41)$ and thus did not meet the recency criteria for APSS and may not have the frequency criteria, and people who reported "vague symptoms" that neither met severity nor frequency for APSS $(n=27)$.

Participants were excluded if they met criteria for any current or lifetime axis I psychotic disorder, prior history of treatment with an antipsychotic, IQ $<$ than 70 , or past or current history of a clinically significant central nervous system disorder that may confound or 
contribute to clinical high risk symptoms. Participants were excluded if they were using antipsychotics at baseline. Furthermore, antipsychotics were not used at any later points in this study.

\section{Measures}

Criteria for a prodromal syndrome and criteria for conversion to psychosis were determined using the Structured Interview for Prodromal Syndromes (SIPS) (McGlashan et al., 2010). Conversion meant that at least one of the five attenuated positive symptoms reached a psychotic level of intensity (rated 6) for a frequency of $\geqslant 1$ hour/day for 4 days/week during the past month or that symptoms seriously impacting functioning (e.g. severely disorganized or dangerous to self or others) (McGlashan et al., 2010). Symptoms were assessed with the Scale of Prodromal Symptoms (SOPS), which consists of 19 items in 4 symptom domains: positive, negative, general, and disorganized. The Structured Clinical Interview for DSM-IV (SCID-I) (First, Spitzer, Gibbon, Williams and Williams, 1995) was used to determine the presence of any axis I disorder.

Metacognition was assessed using the Meta-Cognitions Questionnaire (MCQ) (CartwrightHatton and Wells, 1997), a 65-item scale with each item being rated on a 4-point scale, from 1 (do not agree) to 4 (agree very much). Among all the measures that are available to assess metacognition, the MCQ is the most comprehensive, and has been developed to assess specific dimensions of metacognition that are thought to be involved in the development of psychological disturbances, in accord with the S-REF model. The MCQ has five sub-scales: (i) positive beliefs about worry, which includes items related to the idea that worrying is necessary to solve problems; (ii) negative beliefs about uncontrollability of thoughts and corresponding danger, with items related to beliefs about mental and physical danger of worrying and about worrying being uncontrollable; (iii) cognitive confidence, which refers to the efficacy of one's cognitive skills such as attention and memory; (iv) negative beliefs about thoughts in general, including themes of responsibility, punishment and superstition whose items concern the negative outcomes that might result from specific thoughts; and (iv) cognitive self-consciousness, which includes items regarding one's tendency to focus on their own thinking processes.

\section{Procedures}

All three sites involved in this longitudinal study of predictors of conversion to psychosis recruited CHR and HSC individuals. Raters were experienced research clinicians who demonstrated adequate reliability at routine reliability checks. Gold standard post-training agreement on the critical threshold for determining initial eligibility and subsequent conversion status based on the SIPS was excellent (kappa $=0.90$ ). The PI or clinical psychiatrist or psychologist at each site conducted a comprehensive clinical assessment to determine if entry criteria were met. Inter-rater reliability for the SCID was determined at the start of the study and annually by $100 \%$ agreement on the diagnosis and at least $80 \%$ agreement for symptom presence. Jean Addington chaired weekly conference calls to review criteria for all individuals admitted to the study. The study protocols and informed consents were reviewed and approved by the ethical review boards of all three study sites. All the 
participants in the PREDICT study completed the MCQ. Participants were tested at baseline and followed-up after 6 months using the MCQ.

\section{Statistical analyses}

A logarithmic transformation was applied to the data obtained from the MCQ to comply with the assumption of normality. The Student $t$-test and chi-square test were used to compare baseline demographic differences as well as baseline differences in symptoms between the CHR group and the HSC group. Paired $t$-test was used to examine changes over time in symptoms. Spearman's correlation was used to determine associations amongst the MCQ and the SOPS. To account for any missing data and intra-participant correlation over time, mixed effects models for longitudinal data were used to compare metacognition (MCQ total score and sub-scores) between/within the two groups at baseline and 6-month follow-up. Additional analyses were conducted to compare converters and non-converters from both groups using Mann-Whitney U test given the unequal sample sizes in the two groups.

\section{Results}

\section{Sample characteristics}

No significant differences were observed between the CHR and the HSC groups on any of the demographic variables at baseline. The majority of participants were white $(77 \%)$, male (59\%), never married (96\%) and with a mean age of $19.7(S D=4.2)$ years. Forty-six percent had not completed high school, most likely due to the young age of the sample, and $35 \%$ had graduate degrees or certificates. Compared to the HSC group, the CHR group had significantly higher ratings on SOPS attenuated positive symptoms $(t=6.73, p<.001)$ and on SOPS general symptoms $(t=3.23, p<.01)$. No significant differences were observed in negative or disorganized symptoms between the two groups. Over the first 6 months, participants in both groups improved in SOPS positive and negative symptoms sub-scales (all $p \leqslant .001$ ). Over the course of the PREDICT study, 32 participants (15 males and 17 females) developed psychosis. Since there were a number of dropouts in this study, we compared those who dropped out to those who completed the 6-month assessment on baseline demographics, symptoms and metacognitions. For both groups, no differences were observed between those who completed the 6-month assessment and those who dropped out except for the age in the HSC group, which was slightly higher for those who dropped out $[F(1,27)=7.349, p<.05]$.

\section{Metacognition}

Due to the lack of a healthy control sample in this study, we used data reported in the literature to visually examine performance of our CHR sample within the psychosis continuum. Table 1 presents means and standard errors observed in our study before the logarithmic transformation, and results from psychotic patients and healthy control subjects groups published in the literature (Morrison et al., 2007). In almost all sub-scores, our CHR sample scored lower than psychotic patients from previous studies but slightly higher than nonpatients. 
Table 1. Comparison of baseline performance of clinical high risk and help seeking controls with psychotic patients and healthy volunteers from the literature

\begin{tabular}{|c|c|c|c|c|}
\hline \multirow[b]{2}{*}{ MCQ sub-scores } & \multicolumn{2}{|c|}{ PREDICT groups } & \multicolumn{2}{|c|}{ Morrison et al., 2007} \\
\hline & $\begin{array}{l}\text { CHR mean } \\
\quad(\mathrm{SE}) \\
N=125\end{array}$ & $\begin{array}{l}\text { HSC mean } \\
\quad(\mathrm{SE}) \\
N=64\end{array}$ & $\begin{array}{c}\text { Psychotic patients* } \\
\text { mean (SE) } \\
N=73\end{array}$ & $\begin{array}{c}\text { Non-patients** } \\
\text { mean }(\mathrm{SE}) \\
N=188\end{array}$ \\
\hline Positive beliefs & $33.21(0.98)$ & $30.89(1.05)$ & $40.55(1.38)$ & $32.93(0.81)$ \\
\hline $\begin{array}{l}\text { Negative beliefs about } \\
\text { uncontrollability }\end{array}$ & $38.19(1.13)$ & $32.77(1.43)$ & $44.90(1.42)$ & $28.21(0.83)$ \\
\hline Cognitive confidence & $20.66(0.62)$ & $18.08(0.83)$ & $25.12(0.83)$ & $16.52(0.49)$ \\
\hline Negative beliefs & $26.74(0.70)$ & $22.56(0.79)$ & $32.01(0.84)$ & $20.27(0.49)$ \\
\hline Cognitive self-consciousness & $19.39(0.44)$ & $18.53(0.56)$ & $19.12(0.65)$ & $16.13(0.38)$ \\
\hline
\end{tabular}

Notes: $\mathrm{CHR}=$ Clinical High Risk; HSC $=$ Help-Seeking Controls; MCQ $=$ Meta-Cognitions Questionnaire; ${ }^{*}$ mean age $=41.2(10.3) ;{ }^{* *}$ mean age $=27.6(11.1)$

We checked for correlations between symptoms and MCQ sub-scores. After applying Bonferroni correction there were associations in the CHR group between Negative beliefs about uncontrollability and Cognitive confidence sub-scores and general symptoms (respectively $r_{s}=0.301, p<.001 ; r_{s}=0.260, p<.005$ ).

\section{Comparisons between/within groups}

Results of the mixed effects modeling demonstrated that compared to the HSC $(n=68)$ the CHR ( $n=153$ ) group performed more poorly at baseline on Negative beliefs about uncontrollability, Negative beliefs, and the total MCQ. At the 6-month assessment, no group differences were observed on any of the sub-scores or the total score (CHR: $n=80$, HSC: $n=41)$. These results are presented in Table 2 .

Over the first 6 months of the study the CHR group significantly improved on Negative beliefs about uncontrollability, Cognitive confidence, Negative beliefs, Cognitive selfconsciousness, and the overall MCQ score. The HSC group improved on Negative beliefs about uncontrollability and Cognitive confidence (see Table 2).

Within the first 2 years of the study, 32 participants, $28 \mathrm{CHR}$ and $4 \mathrm{HSC}$, converted to psychosis. We had hypothesized that high scores in positive beliefs about worry would predict conversion to psychosis. A comparison of the converters $(n=28)$ and non-converters $(n=125)$ within the CHR group did not show any difference. Since no differences were observed in positive beliefs at baseline between groups, the CHR and HSC groups were combined in order to compare converters with nonconverters from both groups. In this combined sample of converters $(n=32)$ and nonconverters $(n=189)$ there were significant differences in three (Positive beliefs: $U=$ 2320.50, $p<.05$; Negative beliefs about uncontrollability: $U=2350.00, p<.05$; Negative beliefs: $U=2306.00, p<.05)$ of the five factors and in the total score $(U=2218.00, p<.05)$. No significant differences were observed for Cognitive confidence $(U=2658.50, p=.27)$ and Cognitive self-consciousness $(U=2519.00, p=.13)$. 
Table 2. Comparison between/within groups at baseline and 6-months

\begin{tabular}{|c|c|c|c|c|c|c|c|c|}
\hline \multirow[b]{2}{*}{ MCQ } & \multicolumn{4}{|c|}{$\begin{array}{l}\text { Baseline between groups } \\
\qquad d f=219\end{array}$} & \multicolumn{4}{|c|}{$\begin{array}{l}\text { 6-months between groups } \\
\qquad d f=219\end{array}$} \\
\hline & $t$ & $p$ & $d$ & 95\% C.I. & $t$ & $p$ & $d$ & $95 \%$ C.I. \\
\hline Positive beliefs & -1.79 & 0.28 & 0.24 & $-6.62,1.2$ & -0.69 & 0.90 & 0.09 & $-5.63,3.26$ \\
\hline $\begin{array}{l}\text { Neg. beliefs about } \\
\text { uncontrollability }\end{array}$ & $-\mathbf{3 . 1 1}$ & 0.01 & 0.42 & $-10.39,-0.96$ & -2.06 & 0.17 & 0.28 & $-9.05,1.04$ \\
\hline Cognitive confidence & -2.23 & 0.11 & 0.30 & $-4.9,0.36$ & -2.16 & 0.14 & 0.29 & $-4.89,0.44$ \\
\hline Negative beliefs & -3.8 & 0.001 & 0.51 & $-7.1,-1.35$ & -1.77 & 0.29 & 0.24 & $-5.2,0.97$ \\
\hline $\begin{array}{l}\text { Cognitive self- } \\
\text { consciousness }\end{array}$ & -1.7 & 0.32 & 0.23 & $-2.9,0.6$ & 0.13 & 0.99 & 0.02 & $-2.12,2.35$ \\
\hline \multirow[t]{4}{*}{ Total score } & -3.56 & 0.002 & 0.48 & $-27.7,-4.39$ & -1.96 & 0.21 & 0.26 & $-22.2,3.07$ \\
\hline & \multicolumn{8}{|c|}{ Over time within groups $d f=219$} \\
\hline & \multicolumn{4}{|c|}{ CHR } & \multicolumn{4}{|c|}{ HSC } \\
\hline & $t$ & $p$ & $d$ & 95\% C.I. & $t$ & $p$ & $d$ & 95\% C.I. \\
\hline Positive beliefs & 1.09 & 0.70 & 0.14 & $-1.28,3.01$ & -0.55 & 0.96 & 0.07 & $-3.6,2.3$ \\
\hline $\begin{array}{l}\text { Neg. beliefs about } \\
\text { uncontrollability }\end{array}$ & 6.22 & $<0.0001$ & 0.84 & $3.4,8.3$ & 3.15 & 0.01 & 0.42 & $0.74,7.59$ \\
\hline Cognitive confidence & 3.86 & $<0.001$ & 0.52 & $0.62,3.17$ & 2.65 & 0.04 & 0.36 & $0.04,3.6$ \\
\hline Negative beliefs & 3.71 & 0.001 & 0.50 & $0.70,3.93$ & 0.23 & 0.99 & 0.03 & $-2.08,2.47$ \\
\hline $\begin{array}{l}\text { Cognitive self- } \\
\text { consciousness }\end{array}$ & 2.82 & 0.03 & 0.38 & $0.11,2.48$ & 0.04 & 0.99 & 0.005 & $-1.65,1.71$ \\
\hline Total score & 5.27 & $<0.0001$ & 0.71 & $6.14,17.98$ & 1.74 & 0.30 & 0.23 & $-2.7,13.9$ \\
\hline
\end{tabular}

Notes: CHR $=$ Clinical High Risk; HSC $=$ Help-Seeking Controls; MCQ $=$ Meta-Cognitions Questionnaire

\section{Discussion}

The aim of this study was to determine if negative beliefs about thoughts are characteristic of people at CHR for psychosis and if positive beliefs about worry predict later conversion to psychosis. To our knowledge, this is the first study to longitudinally assess metacognition and its relationship to later conversion to psychosis in this population. Due to the lack of a healthy control group, we cannot state that metacognitive functioning is impaired in CHR individuals. However, a visual examination of data available in the literature seems to suggest that our participants had very similar scores to those in the Brett et al. (2009) study, with lower scores than psychotic patients and higher scores than healthy volunteers. Most previous studies have used healthy controls as the control group. In this study, a sample of young help-seekers was chosen as a control group to determine if the deficit was specific to those at CHR.

Our results showed that the CHR group had higher scores compared to the HSC group in two out of five sub-scales of the MCQ, which concerned negative metacognitive beliefs (negative beliefs about uncontrollability of thoughts and negative beliefs about thoughts in general). This confirms previous literature suggesting that high negative metacognitive beliefs are characteristic of people who meet the clinical high risk criteria (e.g. Brett et al., 2009). Our HSC group consisted mainly of those with long standing symptoms, individuals who were 
not currently meeting prodromal criteria but who most likely met it in the past. At 6-months assessment, no significant differences between groups were observed, a result that can be explained by the improvement over time in symptoms and metacognition that was observed for both groups but was more pronounced for the CHR.

Based on a comparison of psychotic patients and CHR individuals, it was suggested that higher scores (i.e. poorer metacognition) in positive beliefs about worry would predict conversion to psychosis (Morrison et al., 2007). However, in this study, comparison of the converters and non-converters revealed that the converters at baseline had higher scores in the sub-domains of positive beliefs about worry (positive domain), negative beliefs about uncontrollability of thoughts and danger, and negative beliefs about thoughts in general, suggesting that both positive and negative metacognitive beliefs may play a role in the development of psychosis.

There are limitations to this study. First, there was a loss of $45 \%$ of participants at the 6month follow-up assessment. This was due to a dropout rate of $26 \%, 10 \%$ missing because we recruited throughout the study, and some people were not in the study long enough to do the 6-month follow-up, and $9 \%$ had converted. This raises uncertainty as to whether our sample included more potential converters than those who developed psychosis during their time in the study. Nevertheless, we did not observe any baseline differences between those who dropped out and those who stayed in the study. However, even if there were some "future converters" in our non-converting group we still observed a difference. A second limitation is that the lack of a healthy control group does not allow us to determine if metacognition is impaired in the CHR population, although this seems to be established in the literature (e.g. Brett et al., 2009). The strengths of this study are that metacognition was assessed longitudinally in a large sample of individuals at CHR for psychosis, the sample was antipsychotic naïve, and we had a clinical control sample.

In conclusion, our results confirm that CHR individuals are characterized by poorer negative metacognitive beliefs and suggest that a poorer performance on both positive and negative dimensions of metacognition can be considered as a marker of developing psychosis. These results support the potential value of assessing metacognitive beliefs in individuals vulnerable for psychosis as it may be a promising psychological factor worthy of attention in high risk populations. In the literature some success has been reported using CBT approaches with those at CHR (Addington et al., 2011; Morrison et al., 2012) and metacognitive training (MCT) has already proven its efficacy as a complementary intervention in schizophrenia (e.g. Moritz, Veckenstedt, Randjbar, Vitzthum and Woodward, 2011) by reducing specific cognitive biases that are involved in the pathogenesis of positive symptoms.

\section{Acknowledgements}

This work was supported by National Institute of Mental Health grants to J. Addington (grant number U01MH06634-02), to D. Perkins (grant number U01MH066069-04), and to S. Woods (grant number U01MH066160). The National Institute of Mental Health had no further role in study design; in the collection, analysis and interpretation of data; in the writing of the report; and in the decision to submit the paper for publication. 


\section{References}

Addington, J., Epstein, I., Liu, L., French, P., Boydell, K. M. and Zipursky, R. B. (2011). A randomized controlled trial of cognitive behavioral therapy for individuals at clinical high risk of psychosis. Schizophrenia Research, 125, 54-61.

Barkus, E., Stirling, J., French, P., Morrison, A., Bentall, R. and Lewis, S. N. (2010). Distress and metacognition in psychosis prone individuals: comparing high schizotypy to the at-risk mental state. The Journal of Nervous and Mental Disease, 198, 99-104.

Brett, C. M. C., Johns, L. C., Peters, E. P. and McGuire, P. K. (2009). The role of metacognitive beliefs in determining the impact of anomalous experiences: a comparison of help-seeking and nonhelp-seeking groups of people experiencing psychotic-like anomalies. Psychological Medicine, 39, 939-950.

Cartwright-Hatton, S. and Wells, A. (1997). Beliefs about worry and intrusions: the Meta-Cognitions Questionnaire and its correlates. Journal of Anxiety Disorders, 11, 279-296.

First, M., Spitzer, R. L., Gibbon, M., Williams, B. and Williams, J. B. W. (1995). Structured Clinical Interview for DSM-IV Axis I Disorders, Patient Edition. New York: Biometrics Research Department, New York State Psychiatric Institute.

Launay, G. and Slade, P. (1981). The measurement of hallucinatory predisposition in male and female prisoners. Personality and Individual Differences, 2, 221-234.

McGlashan, T., Walsh, B. C. and Woods, S. W. (2010). The Psychosis Risk Syndrome: handbook for diagnosis and follow-up. New York: Oxford University Press.

Moritz, S., Veckenstedt, R., Randjbar, S., Vitzthum, F. and Woodward, T. S. (2011). Antipsychotic treatment beyond antipsychotics: metacognitive intervention for schizophrenia patients improves delusional symptoms. Psychological Medicine, 41, 1823-1832.

Morrison, A. P. (2001). The interpretation of intrusions in psychosis: an integrative cognitive approach to hallucinations and delusions. Behavioural and Cognitive Psychotherapy, 29, 257-276.

Morrison, A. P., Bentall, R. P., French, P., Walford, L., Kilcommons, A., Knight, A., et al. (2002). Randomised controlled trial of early detection and cognitive therapy for preventing transition to psychosis in high-risk individuals. The British Journal of Psychiatry, 181, s78-s84.

Morrison, A. P., French, P., Stewart, S. L. K., Birchwood, M., Fowler, D., Gumley, D. I., et al. (2012). Early detection and intervention evaluation for people at risk of psychosis: multisite randomised controlled trial. British Medical Journal, 344. doi: 10.1136/bmj.e2233

Morrison, A. P., French, P. and Wells, A. (2007). Metacognitive beliefs across the continuum of psychosis: comparisons between patients with psychotic disorders, patients at ultra-high risk and non-patients. Behaviour Research and Therapy, 45, 2241-2246.

Morrison, A. P., Nothard, S., Bowe, S. E. and Wells, A. (2004). Interpretations of voices in patients with hallucinations and non-patient controls: a comparison and predictors of distress in patients. Behaviour Research and Therapy, 42, 1315-1323.

Morrison, A. P. and Wells, A. (2003). A comparison of metacognitions in patients with hallucinations, delusions, panic disorder, and non-patient controls. Behaviour Research and Therapy, 41, 251-256.

Morrison, A. P., Wells, A. and Nothard, S. (2000). Cognitive factors in predisposition to auditory and visual hallucinations. British Journal of Clinical Psychology, 39, 67-78.

Semerari, A., Carcione, A., Dimaggio, G., Falcone, M., Nicolo, G., Procaci, M., et al. (2003). How to evaluate metacognitive function in psychotherapy? The Metacognition assessment scale and its applications. Clinical Psychology and Psychotherapy, 10, 238-261.

Wells, A. and Matthews, G. (1994). Attention and Emotion: a clinical perspective. Hove, UK: Lawrence Erlbaum.

Wells, A. and Matthews, G. (1996). Modelling cognition in emotional disorder: the S-REF model. Behaviour Research and Therapy, 34, 881-888.

Wells, A. and Papageorgiou, C. (1998). Relationships between worry, obsessive-compulsive symptoms and meta-cognitive beliefs. Behaviour Research and Therapy, 36, 899-913. 